This article was published in Engineering Applications of Artificial Intelligence, 38, 24-33, 2015

http://dx.doi.org/10.1016/j.engappai.2014.10.014

\title{
Transferrin surface-modified PLGA nanoparticles- mediated delivery of a proteasome inhibitor to human pancreatic cancer cells
}

Manuela F. Frasco, ${ }^{1}$ Gabriela M. Almeida, ${ }^{2}$ Filipe Santos-Silva, ${ }^{2,3,4}$ Maria do Carmo Pereira, ${ }^{1}$ Manuel A. N. Coelho ${ }^{1}$

1LEPABE, Department of Chemical Engineering, Faculty of Engineering, University of Porto, Rua Dr. Roberto Frias, 4200-465 Porto, Portugal

2 Institute of Molecular Pathology and Immunology of the University of Porto (IPATIMUP), Rua Dr. Roberto Frias, 4200-465 Porto, Portugal

${ }^{3}$ Department of Pathology and Oncology, Medical Faculty, University of Porto, Alameda Prof. Hernâni Monteiro, 4200-319 Porto, Portugal

${ }^{4}$ Eppley Institute for Research in Cancer and Allied Diseases, University of Nebraska Medical Center, Omaha, Nebraska, United States

\section{Abstract}

The aim of this study was to develop a drug delivery system based on poly(lactic-co-glycolic acid) (PLGA) nanoparticles for an efficient and targeted action of the proteasome inhibitor bortezomib against pancreatic cancer cells. The PLGA nanoparticles were formulated with a poloxamer, and further surfacemodified with transferrin for tumor targeting. The nanoparticles were characterized as polymer carriers of bortezomib, and the cellular uptake and growth inhibitory effects were evaluated in pancreatic cells. Cellular internalization of nanoparticles was observed in normal and cancer cells, but with higher uptake by cancer cells. The sustained release of the loaded bortezomib from PLGA nanoparticles showed cytotoxic effects against pancreatic normal and cancer cells. Noteworthy differential cytotoxicity was attained by transferrin surface-modified PLGA nanoparticles since significant cell growth inhibition by delivered bortezomib was only observed in cancer cells. These findings demonstrate that the ligand transferrin enhanced the targeted delivery of bortezomib-loaded PLGA nanoparticles to pancreatic cancer cells. These in vitro results highlight the transferrin surface- modified PLGA nanoparticles as a promising system for targeted delivery of anticancer drugs. 


\section{INTRODUCTION}

The aggressive nature of pancreatic cancer results in failure of current conventional therapies at time of diagnosis, urging the development of novel strategies to achieve improved outcomes. ${ }^{1}$ Nanoparticles (NPs) offer an unprecedented opportunity in rational drug delivery by enhancing therapeutic efficacy and reducing side effects, overcoming major obstacles of using chemotherapeutic agents such as nonspecific biodistribution with consequent doselimiting toxicity and multidrug resistance (MDR). ${ }^{2,3}$

Drug delivery vehicles made of poly(lactic-co-glycolic acid) (PLGA) polymer have been extensively studied for various therapeutic applications. ${ }^{4}$ PLGA NPs have distinguishing features including biocompatibility, stability, high encapsulation of many agents and sustained drug release, and improved biodistribution by passive or active targeting to cancer cells. ${ }^{5}$ Also, NPs may increase the ability of drugs to penetrate tumor microenvironment in effective concentrations and overcome the MDR of solid tumors. 6 Studies have shown that NPs internalized by endocytosis may insert anticancer drugs into cells without triggering drug efflux pumps. ${ }^{7}$ One of the most characterized drug efflux pumps playing a significant role in drug resistance is $\mathrm{P}$ - glycoprotein, a cell-surface glycoprotein member of the ATP- binding cassette (ABC) transporters. 8 Thus, another possibility to overcome MDR includes direct inhibition of $\mathrm{P}$ - glycoprotein by surfactants, such as the nonionic surfactants poloxamers, which are used in the formulation of NPs. ${ }^{2,9}$

The selective toxicity of anticancer therapeutics by destroying cancer cells without affecting normal tissues can be improved by engineering the surface of NPs to display a variety of conjugated ligands. This ligand-targeted therapeutics uses affinity ligands that specifically recognize and interact with cancer cells through binding to molecules or receptors that are either uniquely expressed or overexpressed on targeted cells. ${ }^{10}$ Iron is essential in many bio- chemical processes and its homeostasis is highly controlled. Transferrin (Tf), an iron-binding plasma glycoprotein of approximately $80 \mathrm{kDa}$, is crucial for the cellular transport of iron mediated by cell surface transferrin receptors (TfRs). Cell surface TfRs can bind diferric Tf (holo-Tf) with high affinity and the formed complex is internalized through receptor-mediated endocytosis. ${ }^{11}$ As iron is required to keep rapid proliferation, Tf has been used as a ligand for tumor targeting because the level of expression of TfRs is higher in numerous malignant tissues and correlate with the aggressive or proliferative ability of cancer cells. 12

In eukaryotic cells, the ubiquitin-proteasome system is the main non-lysosomal pathway responsible for selective intracellular degradation of polyubiquitin-tagged proteins. 13 The proteasome pathway is involved in the control of crucial cellular processes, such as modulation of cell cycle and division, signaling, transcription, and apoptosis. ${ }^{14}$ Recently, the proteasome has been identified as a key target for cancer 
therapy, and its inhibition showed to reduce tumor proliferation and to induce apoptosis. ${ }^{15}$ Bortezomib (BTZ) is a boronic acid dipeptide derivative that selectively inhibits the catalytic activity harbored in the $26 \mathrm{~S}$ proteasome, hampering proteasomal functions, and it has been the first proteasome inhibitor tested in clinical trials. ${ }^{14,16}$ In preclinical studies, BTZ decreases cell proliferation, induces apoptosis, sensitizes tumor cells to chemotherapy or radiation and inhibits tumor angiogenesis. ${ }^{16}$ Despite the antitumor activity of BTZ, its dose-limiting toxicity together with resistance development may reduce the effectiveness of treatments.

The aim of this study was to develop a delivery system based on PLGA NPs, which were further surface modified with holo-transferrin (hTf), to effectively incorporate BTZ and to enhance its efficacy and targeted action against pancreatic cancer cells. The NP systems were characterized by various parameters like particle size, stability, and drug release. The delivery of BTZ loaded in the formulated NPs to pancreatic cell lines was evaluated by in vitro cell growth assays.

\section{MATERIALS AND METHODS}

\section{Chemicals}

Poly (D,L-lactide-co-glycolide) (PLGA, Resomer ${ }^{\circledR}$ RG 503 H, lactide:glycolide 50:50, Mw 24,000-38,000), Pluronic ${ }^{\circledR}$ F127 (PF127), ethyl acetate, $N$-SuccinylLeu-Leu-Val-Tyr-7-Amido- 4-Methylcoumarin (Suc-LLVY-AMC), human holotransferrin (hTf), $o$-phthaldialdehyde (OPA) reagent solution (complete), sulforhodamine B (SRB), coumarin-6 (C6), propidium iodide and trypan blue were purchased from Sigma-Aldrich (St. Louis, MO, USA). Bortezomib (BTZ) was acquired from Sell- eck Chemicals (Houston, TX, USA). Purified 20S proteasome (human erythrocyte) was purchased from Enzo Life Sciences. (Farmingdale, NY, USA). Dulbecco's modified Eagle medium (DMEM) (with low glucose, pyruvate, HEPES), phosphate buffered saline (PBS), trypsin and PrestoBlue ${ }^{\circledR}(\mathrm{PB})$ cell viability reagent were obtained from Life Technologies (Carlsbad, CA, USA). Fetal bovine serum (FBS) was acquired from Biowest SAS (Nuaill'e, France). All other chemicals were of analytical grade.

\section{Cell lines}

The two human pancreatic cell lines, hTERT-HPNE (Human Pancreatic NestinExpressing (HPNE) cells are normal duct- derived cells of the human pancreas immortalized with the catalytic subunit of telomerase (hTERT)) and S2-013 (well differentiated tubular adenocarcinoma and moderately met- astatic subline cloned from the human pancreatic tumor cell line SUIT-2) were kindly provided by Prof. M. A. Hollings- worth (Eppley Institute for Research in Cancer and Allied Diseases, UNMC, Omaha, NE, USA). 17,18 Adherent cell cultures were maintained in antibiotic-free DMEM with $10 \%$ heat-inactivated $\mathrm{FBS}$ at $37^{\circ} \mathrm{C}$ in a humidified atmosphere of $5 \% \mathrm{CO} 2$ incubator. 


\section{Formulation of PLGA nanoparticles}

PLGA NPs were formulated by a single-emulsion solvent evaporation technique. PLGA (10 mg) was dissolved in ethyl acetate $(0.1 \mathrm{~mL})$. An aqueous solution of $1 \%$ (w/v) Pluronic ${ }^{\circledR}$ F127 (PF127) (0.2 mL) was added dropwise and the emulsion obtained by sonication $(60 \mathrm{~s})$. This mixture was poured into $2.5 \mathrm{~mL}$ of $0.1 \%(\mathrm{w} / \mathrm{v})$ PF127 and stirred $(800 \mathrm{rpm})$ at room temperature for $3 \mathrm{~h}$. The suspension was filtered $(0.2 \mathrm{~mm})$ and the NPs were collected by centrif- ugation $(14,100 \mathrm{~g}, 30 \mathrm{~min})$ and resuspended in ultrapure water. PLGA NPs unloaded or loaded with BTZ (1 mg) were further modified with hTf by adsorption. The PLGA NPs were incubated overnight with hTf $(1 \mathrm{mg} / \mathrm{mL})$, centrifuged $(14,100 \mathrm{~g}, 30 \mathrm{~min})$, and resuspended in ultrapure water.

\section{Characterization of PLGA nanoparticles}

For determination of particle size and surface charge ( $\zeta$ potential), the NP suspensions were analyzed using a Zeta- sizer Nano ZS (Malvern Instruments Ltd., Malvern, UK). The measurement of the NPs size was based upon photon correlation spectroscopy. This technique yields the hydrodynamic diameter, the particle diameter plus the thickness of the sur- rounding hydration layer, and the polydispersity index (PDI), which is a dimensionless measure for the broadness

of the particle size distribution. The $\zeta$ potential was analyzed based on laser Doppler velocimetry using the same instrument. All measurements were performed at $37^{\circ} \mathrm{C}$. Unloaded PLGA NPs were also analyzed by Transmission Electron Microscopy (TEM) using a JEOL JEM-1400 (JEOL Ltd., Tokyo, Japan) operated at an accelerating voltage of $80 \mathrm{kV}$. The suspension of NPs was deposited for 5 min on 400 mesh Formvar-coated copper TEM grids (Agar Scientific, UK) followed by negative staining $(45 \mathrm{~s})$ with $2 \%(\mathrm{w} / \mathrm{v})$ uranyl acetate solution. The excess solution was blotted off using filter paper and the grids were air dried before TEM imaging. The images were acquired with a SC1000 ORIUS ${ }^{\circledR}$ CCD camera (Gatan Inc., Warrendale, PA, USA). The PLGA- hTf NP suspensions were assayed for protein content using o-phthaldialdehyde (OPA) reagent, which in the presence of reduced sulfhydryl groups reacts with primary amines yielding a fluorescent product. Briefly, samples incubated with OPA were analyzed in 96-well plates by fluorescence at excitation and emission wavelengths of 340 and $460 \mathrm{~nm}$, respectively (Synergy 2 Multi-Mode Microplate Reader, Bio- Tek Instruments Inc., Winooski, VT, USA). The total amount of hTf was determined using a standard curve of known transferrin concentrations. The results are presented as mean and standard deviation (SD) of at least three independent experiments. The adsorption of hTf on PLGA surface was further investigated using Attenuated Total Reflectance (ATR)-Fourier Transform Infrared (FT-IR) spectroscopy. The ATR-FT-IR spectra were recorded using a Bruker spectrometer Alpha (Bruker Optics Inc., Billerica, MA, USA) equipped with a platinum diamond crystal and a DTGS detector at a resolution of $4 \mathrm{~cm}^{-1}$. 


\section{BTZ entrapment efficiency and release from PLGA nanoparticles}

The NP suspensions were centrifuged $(14,100 \mathrm{~g}, 30 \mathrm{~min})$ and the supernatants were collected to determine BTZ entrapment efficiencies by measuring its absorption at

270 nm (Shimadzu UV-1700 spectrophotometer, Shimadzu Corp., Kyoto, Japan). A standard curve of known BTZ concentrations was prepared from a stock solution of BTZ in $5 \%(\mathrm{v} / \mathrm{v})$ ethyl acetate. The results are presented as mean and SD of at least three independent experiments. For the drug release studies, aliquots of suspensions of BTZ loaded in PLGA and PLGA-hTf NPs were diluted in PBS $(\mathrm{pH}$ 7.4) and were incubated over 7 days at $37^{\circ} \mathrm{C}$. At the set time points, the NPs (or degradation products) were separated from the released drug by filtering with $3 \mathrm{kDa}$ molecular weight cutoff (MWCO) Amicon Ultra-0.5 mL Centrifugal Filters (Merck Millipore, Germany) and the kinetic analysis of the 20S proteasome inhibition was performed. The proteasome activity was assessed by monitoring the hydrolysis of the chymotrypsin-like substrate Suc-LLVY-AMC $(37.5 \mathrm{~m} M)$ by the action of the human erythrocyte $20 \mathrm{~S}$ proteasome $(2.5 \mathrm{mg} / \mathrm{mL})$ in proteasome assay buffer (50 $\mathrm{m} M$ Tris/HCl, $25 \mathrm{~m} M \mathrm{KCl}, 10 \mathrm{~m} M \mathrm{NaCl}, 1 \mathrm{~m} M \mathrm{MgCl} 2, \mathrm{pH}$ 7.5). Reaction progress was monitored by the increase in fluorescence (release of free AMC fluorophore, excitation and emission wavelengths of 360 and $460 \mathrm{~nm}$, respectively) at $37^{\circ} \mathrm{C}$ in a microplate reader (Synergy 2 Multi-Mode Microplate Reader, BioTek Instruments Inc., Winooski, VT, USA).

\section{Cellular uptake of PLGA nanoparticles}

Flow Cytometry (FCM) was used to study the cell- association and uptake of PLGA NPs. The lipophilic fluorescent dye coumarin-6 (C6) remains in the polymer matrix of the NPs and does not leach during the experiment allowing a fluorescent visualization of the uptake of PLGA NPs. ${ }^{19}$ The NPs were synthesized as described above and loaded with $\mathrm{C} 6(1 \mathrm{mg})$. Cells were seeded at a density of $1 \times 10^{5}$ cells/ $\mathrm{mL} 48 \mathrm{~h}$ prior to the experiment and then incubated with 100-fold excess of C6-PLGA or C6-PLGA-hTf NPs for 3 h. After incubation with the NPs, cells were washed, pelleted, and resuspended in PBS ( $\mathrm{pH}$ 7.4). Cells were then stained with propidium iodide immediately before flow cytometry (FACSCalibur, BD Biosciences, San Jose, CA, USA). Three independent experiments were carried out, including untreated controls. FCM was performed plotting at least 30,000 events per sample and data were subsequently analyzed by FlowJo 7.2 software (Tree Star, Ashland, USA).

\section{Evaluation of in vitro cytotoxicity}

The effects of BTZ alone and BTZ-loaded NPs on the cell growth of pancreatic cell lines were analyzed by PrestoBlue ${ }^{\circledR}(\mathrm{PB})$ and sulforhodamine B (SRB) assays. Both assays provide an indirect estimation of cell number, the $\mathrm{PB}$ reagent is reduced by metabolically active cells, and the protein-binding dye SRB stains the cellular protein content. Exponentially growing cells were seeded at a density of $1 \times 10^{4}$ cells $/ \mathrm{mL}$ and allowed to 
adhere for $24 \mathrm{~h}$. Cells were then incubated for $48 \mathrm{~h}$ with samples of BTZ alone (0.1 to $100 \mathrm{n} M$ ), and unloaded or BTZ-loaded unmodified and hTf-modified NPs (stock solutions of $0.1 \mathrm{mg} / \mathrm{mL}$ of NPs) diluted in growth medium. Following the $48 \mathrm{~h}$ incubation period, the plates were washed with PBS ( $\mathrm{pH}$ 7.4) and assayed for cell growth using PB according to the manufacturer's instructions. During incubation with the cells, PB reagent is modified by the reducing environment of the viable cells and becomes highly fluorescent. Fluorescence was measured using a microplate reader (Synergy HT MultiMode Microplate Reader, BioTek Instruments Inc., Winooski, VT, USA) at excitation and emission wavelengths of 560 and $590 \mathrm{~nm}$, respectively. For the SRB assay, ${ }^{20}$ cells were fixed with $10 \%(\mathrm{w} / \mathrm{v})$ trichloroacetic acid for $60 \mathrm{~min}$ at $4{ }^{\circ} \mathrm{C}$, the plates were washed with water, dried, and the protein content stained with SRB solution. The unbound dye was removed by washing with $1 \%(\mathrm{v} / \mathrm{v})$ acetic acid, the plates were dried, incorporated dye was solubilized with $10 \mathrm{~m} M$ Tris base buffer and the optical density was measured at $560 \mathrm{~nm}$ using a microplate reader (Synergy HT Multi-Mode Microplate Reader, BioTek

Instruments Inc., Winooski, VT, USA). For all the assays, a mea- sure of the cell density at time zero (i.e., the time at which BTZ/ NPs were added) from extra reference plates was performed. No-treatment control cells were included in all assays and all samples were measured in triplicate in at least three independent experiments. By comparing the measured fluorescence/ absorbance of the wells containing drug or NPs with the measurements of the wells containing untreated cells, following the $48 \mathrm{~h}$ incubation period, and subsequently comparing these results with the ones obtained for cells that had been fixed at time zero (time at which BTZ/NPs were added), it was possible to generate dose-response profiles and determine GI50 (the con- centration that inhibits growth in $50 \%$ ) values.

\section{RESULTS}

\section{Characterization of PLGA nanoparticles}

The preparation of PLGA NPs involved a formulation with PLGA as core polymer and the Pluronic ${ }^{\circledR}$ F127 (PF127, also known as poloxamer 407) as emulsifier, and their narrow size distribution (PDI 0.06) indicates the formation of uniform structures. The monodisperse PLGA NPs were in the nanometer range (192-195 nm) and the $\zeta$ potential was approximately $-27 \mathrm{mV}$ (Table I). These results are in agreement with the size and morphology observed by TEM (Fig. 1) that showed PLGA NPs spherical in shape and with an average diameter of approximately $200 \mathrm{~nm}$. The NPs further surface-modified with hTf by adsorption, formed monodisperse stable PLGA-hTf NPs (PDI < 0.1) (Table I). It was observed a small but not significant increase in the hydrodynamic diameters due to the adsorbed hTf molecular layer without major changes in the PDI. Additionally, the modified NPs showed a significant change in the $\mathrm{f}$ potential that was reduced (in absolute value) to the measured value of approximately $-15 \mathrm{mV}$. The amount of hTf adsorbed to NPs was $49 \pm 7 \mu \mathrm{g} / \mathrm{mg}$ NPs. The ATR-FT-IR spectrum of unmodified PLGA NPs showed characteristic bands, namely the carbonyl $\mathrm{C} 5 \mathrm{O}$ stretching $\left(1758 \mathrm{~cm}^{-1}\right)$ and the ester C-O stretching (1000- 
$1300 \mathrm{~cm}^{-1}$; the $\mathrm{C}-\mathrm{O}-\mathrm{C}$ stretching of $\mathrm{PEO} / \mathrm{PPO}$ chains of PF127 is also included in this region) (Fig. 2). ${ }^{21}$ In the spectrum of the protein hTf, two main peaks appeared at $1648 \mathrm{~cm}^{-1}$ and $1543 \mathrm{~cm}^{-1}$ that can be assigned to the amide I and amide II vibrations, respectively (Fig. 2). The spectrum of PLGA NPs coated with hTf showed the amide bands assigned to the protein. The peak of the amide I band in the spectrum of PLGA-hTf NPs occurred at a slightly higher frequency $\left(1655 \mathrm{~cm}^{-1}\right)$. In addition, the bands of the characteristic functional groups of PLGA NPs seem to present small shifts when compared to the spec- trum of unmodified NPs (Fig. 2).

\section{BTZ entrapment efficiency and release from PLGA nanoparticles}

The BTZ entrapment efficiency, expressed as the percentage of drug amount in the PLGA NPs to the amount of drug fed initially, was found to be $53 \pm 6 \%(\mathrm{w} / \mathrm{w})$. Controlled release of BTZ occurred by an initial burst (over the first few hours) followed by a slow sustained release for days (Fig. 3). By comparing the release profiles of BTZ, it was observed a higher release from unmodified PLGA in comparison to PLGA-hTf NPs (Fig. 3).

\section{Cellular uptake of PLGA nanoparticles}

Pancreatic cells incubated with PLGA NPs or with PLGA-hTf NPs both encapsulating C6 (their physicochemical proper- ties were similar to those described for unloaded NPs) were analyzed through FCM to evaluate the targeting ability of hTf-modified NPs. Cell-associated and internalized C6-PLGA NPs were observed in both cell lines. A significant higher fluorescence signal was detected in S2-013 cancer cells, with around $50 \%$ of live cells showing fluorescence due to C6-PLGA NPs [Fig. 4(B)], in comparison to the observed fluorescence in about $10 \%$ of hTERTHPNE normal cells [Fig. 4(A)]. In vitro proliferating cells are expected to express TfRs, and as suggested by FCM, C6-PLGA-hTf NPs uptake occurs by both cell lines (Fig. 4). Nonetheless, the presence of hTf does not seem to significantly improve the cell- associated and internalized NPs in hTERT-HPNE cells, with an increase of about 6\% in cells showing fluorescence due to NPs [Fig. 4(A)]. The active targeting may be more relevant in the S2-013 cancer cells (an increase of about 15\%) in addition to the already observed higher amount of unmodified PLGA NPs associated/internalized in these cells [Fig. 4(B)].

\section{Assessment of cytotoxic effects on pancreatic cells}

As shown in Figure 5, the cytotoxic effects of BTZ alone to both cell lines were evaluated and the GI50 values deter- mined. The GI50 values attained in the $\mathrm{n} M$ range suggest that BTZ is effective at inhibiting cell growth, showing similar toxicity to both cell lines (Table II). Based upon the encapsulation efficiency and the in vitro release profiles, the concentration range of NPs assayed was chosen to deliver similar intracellular BTZ concentrations at $48 \mathrm{~h}$ as in the tested free BTZ. The unloaded PLGA NPs formulated with PF127 were also studied in terms of potential cytotoxicity. The results suggest the absence of adverse effects, with no 
significant concentration-related changes on hTERT-HPNE cell growth observed after cell incubation for $48 \mathrm{~h}$ in the tested concentration ranges. The adsorption of hTf on the surface of the NPs showed to keep the properties described above of being well-tolerated by these cells (Fig. 6 and Sup- porting Information Figure S1). Similar results were obtained with S2-013 cancer cells (Fig. 7 and Supporting Information Figure S2). The cytotoxicity assays confirmed that the unmodified BTZ-loaded NPs provided therapeutically active concentrations of BTZ within S2-013 cells (Fig. 7 and Supporting Information Figure S2). Cytotoxic effects were also observed in the hTERT-HPNE cells (Fig. 6 and Supporting Information Figure S1), though the effect was slightly less pronounced in comparison to S2-013 cells. The concentrations of BTZ inhibiting cell growth in 50\% (GI50 values) were estimated as $7.2 \pm 1.0 \mathrm{n} M$ and $5.8 \pm 1.1 \mathrm{n} M$ for hTERT-HPNE and S2-013 cells, respectively. This result suggests some selectivity of PLGA NPs to cancer cells with lower toxicity to normal cells. A comparison between BTZ- encapsulated in modified and unmodified PLGA NPs revealed the effectiveness of PLGA-hTf NPs in targeting cancer cells. The efficacy was observed by affecting growth of S2-013 cells (Fig. 7 and Supporting Information Figure S2) with a GI50 value of $7.4 \pm 0.5 \mathrm{n} M$ of BTZ and with a significant reduced effect in hTERT-HPNE cells (Fig. 6 and Supporting Information Figure S1), with cell growth above $80 \%$ in comparison to untreated controls. The GI50 values were determined according to the tested concentrations of NPs and the in vitro release profiles at $48 \mathrm{~h}$ of the assay. These results are in agreement with a lower release rate of BTZ from PLGA-hTf NPs together with a non-significant improved uptake of these targeted NPs by hTERT-HPNE cells [Figs. 3 and 4(A)]. The cytotoxicity data obtained for S2-013 cells suggest that the improvement in the hTf- mediated cellular internalization [Fig. 4(B)] is advantageous for the efficacy of actively targeted PLGA NPs (Fig. 7 and Supporting Information Figure S2), despite a slower BTZ release when compared to unmodified NPs.

\section{DISCUSSION}

The obtained monodisperse and stable PLGA NPs, formed in the presence of Pluronic $^{\circledR}$ F127 (PF127), are desired to ensure efficiency of targeting and drug delivery. Poloxamers (known by the trade name Pluronic ${ }^{\circledR}$ ) are nonionic triblock copolymers and consist of hydrophilic poly(ethylene oxide) (PEO) and hydrophobic poly(propylene oxide) (PPO) moieties arranged in ABA structure, i.e., in PEO-PPOPEO, exhibiting amphiphilic characteristics. ${ }^{22}$ In the current formulation with PF127, the PPO unit will interact towards the hydrophobic core of the NPs, while the PEO blocks are extended to the aqueous dispersant. The PLGA NPs achieve colloidal stability from the electric charge of the carboxyl groups of the PLGA, creating a surface charge density. Also, a variety of stability mechanisms depending on the PF127 coverage may contribute for the small sized NPs to be highly stable. ${ }^{23}$ Protein adsorption on biomaterial surfaces is a complex process as it can involve multiple non-covalent 
interactions, such as hydrogen bonding, van der Waals, hydrophobic and electrostatic. $^{24}$ The adsorption of hTf on PLGA NPs occurs through a combination of intermolecular forces that are reflected in the f potential changes. Namely, the hTf layer partially screens the surface charge of the NPs reducing the $\zeta$ potential values. Measuring the increase in the hydrodynamic diameter of 200 nm PLGA NPs upon hTf (hydrodynamic diameter of $\sim 7 \mathrm{~nm}$ ) adsorption corresponds to a change of only $7 \%$, and the size determination may not be feasible to infer on the protein corona. 25 Thus, concurrently results on hTf quantification and ATR-FT-IR spectroscopy suggest that PLGA NPs were effectively surface-modified. In the spectrum of PLGA-hTf NPs, slightly shifts were observed in the bands of the characteristic functional groups of PLGA NPs, possible due to intermolecular interactions with hTf. Also, the amide bands of the hTf in the spectrum of PLGAhTf NPs showed no significant alteration in their positions in comparison to the free protein spectrum, despite the observed small shift in the amide I peak. The amide I band is useful to analyze the secondary structure content of a protein since it is the most sensitive to any conformational changes. ${ }^{26}$ This result suggests a small rearrangement of the hTf structure due to flexibility requirements upon adsorption on the surface of the NPs without major changes in the structural conformation. Thus, the characterization indicates the formation of stable, well- dispersed unmodified and hTf surface modified PLGA NPs. BTZ was efficiently incorporated into PLGA NPs and its sustained release, subsequently studied by in vitro release experiments, was followed over several days. Drug release from PLGA polymers occurs through a combination of degradation (hydrolysis of the ester bonds) and erosion of the polymer (the formation of pores allows the release of oligomers and monomers) and since degradation of PLGA is slow, it will depend mainly on drug diffusion and matrix erosion. As hydrolysis proceeds, the accumulated acidic breakdown products, which remain entrapped in the matrix, control the low $\mathrm{pH}$ in the core, autocatalyzing the process and resulting in the acceleration of degradation. 27 The physicochemical properties of the incorporated drug and its interaction with the polymeric matrix are also known to influence drug release. ${ }^{28}$ One disadvantage of PLGA NPs could be the acidic environment created upon hydrolysis of the ester bonds that might damage loaded BTZ. In this study, the released BTZ was analyzed through the inhibition of the proteasome. Since BTZ maintained its ability to inhibit the proteasome as it was gradually released, the results suggest that the loaded BTZ kept stable inside the polymer matrix. The obtained results with lower release rate of BTZ from PLGA-hTf NPs may be related to a decrease in the hydration rate of the NPs surface. The layer of hTf on the surface of PLGA NPs may hinder water permeation, resulting in a lower diffusion rate of the drug. Similar results have been described when the surface of NPs was modified by adsorption of a monoclonal antibody. 29

BTZ inhibits proliferation of human pancreatic cancer cells, arrests cell cycle progression, induces apoptosis and sensitizes tumor cells to conventional 
chemotherapeutic agents. ${ }^{30,31}$ It has also been reported that BTZ inhibits the growth of human pancreatic tumors by direct induction of tumor cell death and indirect effects on tumor vasculature. The antiangiogenic properties of BTZ is valuable even in tumors that are refractory to the direct cytotoxic effects of the drug, and studies in human pancreatic tumor xenograft models showed that BTZ in combination therapy enhances the antitumor effects of taxanes. ${ }^{32,33}$ Moreover, pancreatic cells are secretory cells with a highly developed endoplasmic reticulum (ER), and proteasome inhibitors such as BTZ may induce ER stress. Selective proteotoxicity would be achieved by targeting the ER through protein aggregation and subsequent ER stress-induced apoptosis. ${ }^{34}$ Data acquired across a cell line panel derived from a wide variety of human tumors (though not including human pancreatic cancer) from the National Cancer Institute (NCI) revealed an average GI50 of $3.9 \mathrm{n} M$ of BTZ. ${ }^{35}$ Other studies using several pancreatic cell lines showed marked heterogeneity in BTZ responsiveness, with some cell lines highly sensitive $(\mathrm{IC} 50<40 \mathrm{n} M)$, while others showed to be resistant $($ IC50 $>10 \mu M) .{ }^{32}$ The results obtained herein suggest that the cell lines tested are very sensitive to BTZ inhibitory effects.

It has been referred that BTZ is less active against solid tumors and has significant dose-limiting toxicity. Other factors contribute to its reduced efficacy, including nonspecific binding to proteins and rapid hepatic clearance. Regarding these limitations, PLGA NPs as delivery vehicles may improve the unfavorable pharmacokinetic properties of BTZ. In terms of cytotoxicity of unloaded PLGA NPs, the results obtained in this study are in accordance with the literature, as PLGA is well tolerated by cells, with known very limited cytotoxicity and an absence of inflammatory responses. ${ }^{36}$ Reported studies on free surfactant cytotoxicity indicate that Pluronic ${ }^{\circledR}$ F127 (PF127, known as poloxamer 407) used in the current study is the most cytocompatible among some other poloxamers, showing low toxicity and immunogenicity. ${ }^{37}$ After the internalization of PLGA NPs by endocytosis, the surface charge of PLGA NPs in the acidic environment of late endosomes changes from anionic to cat- ionic, resulting in their interaction with the membrane and subsequent escape into the cytosol. ${ }^{19}$ A fraction of NPs from the early endosomes is also expected to be sorted to recycling endosomes that recycle them back to the cell surface, and also to be routed along the degradation pathway to lysosomes. ${ }^{38}$ As cytoplasmic delivery vehicles, the endolysosomal escape of PLGA NPs is crucial to achieve a sustained therapeutic effect. The differences in the GI50 values observed between free BTZ and the BTZ-loaded NPs can be related to the kinetics of BTZ release from NPs, and also to differences of cellular uptake pathways since free drugs enter the cells via passive diffusion and NPs are internalized by endocytosis.

The NPs can pass through leaky and hyperpermeable tumor vasculature and together with decreased lymphatic drainage allow the NPs to accumulate in the 
tumor vicinity. 6 This enhanced permeability and retention (EPR) effect is a guiding principle in designing cancer-targeting nanocarriers. ${ }^{5}$ In the case of hypovascular tumors such as pancreatic cancer, active targeting can increase the potential for drug delivery and involves tagging the nanocarrier with a tissue- or cell-specific ligand. The ligand is chosen to bind specific receptors overexpressed in tumor cells, such as the TfRs. ${ }^{6}$ Here, hTf was used to modify the surface of PLGA NPs for the delivery of BTZ. The specificity of the protein coating implies that the mechanisms of endocytosis of PLGA NPs are governed by hTf cellular uptake, involving the binding of hTf to its receptor, and their internalization through receptor-mediated endocytosis. ${ }^{12}$ Rapidly dividing cells like cancer cells express higher TfR levels than their normal tis- sue counterparts. Moreover, the number of surface TfRs has also been shown to be elevated in dividing cells in vitro. ${ }^{39}$ However, not only differences in the expression of cell surface TfRs and in their functional role, but also alterations in the intracellular cycling of the transferrin-receptor conjugates, including rates of endocytosis and exocytosis, may affect the intracellular retention of drugs slowly released by nanocarriers that targeted TfRs. ${ }^{12,39}$ In the normal cells studied, the different efficacy between unmodified and PLGA-hTf NPs may be attributed to differential characteristics, such as TfR numbers, efficiency and cycling rates. Thus, the uptake mediated by TfRs and intracellular pathways of hTf-modified NPs require further investigation. Nonetheless, the in vitro cytotoxic effects on pancreatic cancer cells revealed the ability of PLGA NPs for the slow controlled release of BTZ together with the active hTf targeting, keeping the effective concentrations of BTZ in the $<10 \mathrm{n} M$ range.

Adsorption of hTf on the surface of PLGA NPs for targeting cell surface TfRs was successfully attained and shown to direct the NPs towards cancer cells in vitro. The NP surface is relevant in a biological environment as it can define the interaction with other biomolecules, and the biodistribution and clearance of the NPs. A study on Tf adsorbed on polystyrene NPs revealed that upon competitive binding of plasma proteins, the first mono- layer of adsorbed Tf (hard corona) is stable and may be considered irreversibly bound, while the secondary layer (soft corona) exhibits dynamic exchange forming a reversible bound protein layer. ${ }^{40} \mathrm{Also}$, the kinetics of protein exchange and the plasma protein adsorption may depend on the NP. Factors such as differences in size (surface curvature) or functional moieties in NP coatings (e.g., PEG chain length and density) influence the displayed affinity to certain proteins and may be relevant in a given biological environment of the exposure route. ${ }^{41}$ In a study on the behavior of Tftargeted NPs in vivo, it was shown that the organ distribution did not depend on Tf, while the NPs were actively targeted by Tf to the cancer cells within solid tumors. In addition, the active targeting was influenced by Tf content on the NP surface, suggesting a content threshold for achieving effective targeting. ${ }^{42}$ Fail in the efficacy of targeted NPs may also be the outcome of rearrangement and formation of a multiprotein corona in the NPs that shield Tf from recognition by TfRs and originate loss of 
specificity in the active targeting. ${ }^{43}$ Indeed, when NPs are presented to a cell, the interaction reflects the outermost layer of the NP, and the efficiency of targeted-NPs as drug carriers depends on their optimal design pursuing stable functional protein coating and reduced nonspecific binding to be more resilient in their targeting capability.

In conclusion, future work on the behavior and efficacy of the formulated NPs in biological milieu, together with mechanisms of uptake and subcellular sorting in pancreatic cells, may contribute to study the factors influencing NPs targeting the relevant pathways. Also, it would be interesting to address the ability of the proposed NPs to overcome drug resistance. Overall, the results obtained in targeting cancer cells in vitro with PLGA NPs surface-modified with hTf, encourage future in vivo studies where the efficacy of these NPs in improving drug delivery to pancreatic cancer, and the possibility to be potentiated under physiological conditions of tumor microenvironment and hypoxia, could be demonstrated.

\section{REFERENCES}

[1] McCarroll J, Teo J, Boyer C, Goldstein D, Kavallaris M, Phillips PA. Potential applications of nanotechnology for the diagnosis and treatment of pancreatic cancer. Front Physiol 2014;5:2.

[2] Dong X, Mumper RJ. Nanomedicinal strategies to treat multidrugresistant tumors: Current progress. Nanomedicine 2010;5:597-615.

[3] Zhang X-Q, Xu X, Bertrand N, Pridgen E, Swami A, Farokhzad OC. Interactions of nanomaterials and biological systems: Implications to personalized nanomedicine. Adv Drug Deliv Rev 2012;64:1363- 1384.

[4] Makadia HK, Siegel SJ. Poly lactic-co-glycolic acid (PLGA) as biodegradable controlled drug delivery carrier. Polymers 2011;3: 1377-1397.

[5] Danhier F, Ansorena E, Silva JM, Coco R, Le Breton A, Préat V. PLGA-based nanoparticles: An overview of biomedical applica- tions. J Control Release 2012;161:505-522.

[6] Danhier F, Feron O, Préat V. To exploit the tumor microenvironment: Passive and active tumor targeting of nanocarriers for anticancer drug delivery. J Control Release 2010;148:135-146.

[7] Wang Y, Guo M, Lu Y, Ding L-Y, Ron W-T, Liu Y-Q, Song F-F, Yu SQ. Alpha-tocopheryl polyethylene glycol succinate-emulsified poly(lactic- co $^{-}$ glycolic acid) nanoparticles for reversal of multidrug resistance in vitro. Nanotechnology 2012;23:495103.

[8] Szakả cs G, Paterson JK, Ludwig JA, Booth-Genthe C, Gottesman MM. Targeting multidrug resistance in cancer. Nat Rev Drug Dis- cov 2006;5:219-234.

[9] Kabanov AV, Batrakova EV, Alakhov VYu. Pluronic VR block copolymers for overcoming drug resistance in cancer. Adv Drug Deliv Rev 2002;54:759-779.

[10] Bertrand N, Wu J, Xu X, Kamaly N, Farokhzad OC. Cancer nanotechnology: The impact of passive and active targeting in the era of modern 
cancer biology. Adv Drug Deliv Rev 2014;66:2-25.

[11] Qian ZM, Li H, Sun H, Ho K. Targeted drug delivery via the transferrin receptor-mediated endocytosis pathway. Pharmacol Rev 2002;54:561-587.

[12] Daniels TR, Bernabeu E, Rodr' iguez JA, Patel S, Kozman M, Chiappetta DA, Holler E, Ljubimova JY, Helguera G, Penichet ML. The transferrin receptor and the targeted delivery of therapeutic agents against cancer. Biochim Biophys Acta 2012;1820:291-317.

[13] Schmidt M, Finley D. Regulation of proteasome activity in health and disease. Biochim Biophys Acta 2014;1843:13-25.

[14] Adams J. The proteasome: Structure, function, and role in the cell. Cancer Treat Rev 2003;29:3-9.

[15] Wu WKK, Cho CH, Lee CW, Wu K, Fan D, Yu J, Sung JJY. Proteasome inhibition: A new therapeutic strategy to cancer treatment. Cancer Lett 2010;293:15-22.

[16] Adams J. The proteasome: A suitable antineoplastic target. Nat Rev Cancer 2004;4:349-360.

[17] Lee KM, Yasuda H, Hollingsworth MA, Ouellette MM. Notch2positive progenitors with the intrinsic ability to give rise to pancreatic ductal cells. Lab Invest 2005;85:1003-1012.

[18] Iwamura T, Caffrey TC, Kitamura N, Yamanari H, Setoguchi T, Hollingsworth MA. P-selectin expression in a metastatic pancreatic tumor cell line (SUIT-2). Cancer Res 1997;57:1206-1212.

[19] Panyam J, Zhou W-Z, Prabha S, Sahoo SK, Labhasetwar V. Rapid endolysosomal escape of poly(DL-lactide- $c^{-}$-glycolide) nanoparticles: Implications for drug and gene delivery. FASEB J 2002;16: 1217-1226.

[20] Vichai V, Kirtikara K. Sulforhodamine B colorimetric assay for cytotoxicity screening. Nat Protoc 2006;1:1112-1116.

[21] Tob' ıо M, Nolley J, Guo Y, McIver J, Alonso MJ. A novel system based on a Poloxamer/PLGA blend as a tetanus toxoid delivery vehicle. Pharm Res 1999;16:682-688.

[22] Batrakova EV, Kabanov AV. Pluronic block copolymers: Evolution of drug delivery concept from inert nanocarriers to biological response modifiers. J Control Release 2008;130:98-106.

[23] Santander-Ortega MJ, Jó dar-Reyes AB, Csaba N, Bastos-Gonzả lez D, Ortega-Vinuesa JL. Colloidal stability of Pluronic F68-coated PLGA nanoparticles: A variety of stabilisation mechanisms. J Colloid Interface Sci 2006;302:522529.

[24] Vasita R, Katti DS. Structural and functional characterization of proteins adsorbed on hydrophilized polylactide- ${ }^{-} o^{-}$glycolide microfibers. Int $\mathbf{J}$ Nanomedicine 2012;7:61-71.

[25] del Pino P, Pelaz B, Zhang Q, Maffre P, Nienhaus GU, Parak WJ. Protein corona formation around nanoparticles-From the past to the future. Mater Horiz 2014;1:301-313.

[26] Barth A, Zscherp C. What vibrations tell us about proteins. Q Rev 
Biophys 2002;35:369-430.

[27] Gøpferich A. Mechanisms of polymer degradation and erosion. Biomaterials 1996;17:103-114.

[28] Versypt ANF, Pack DW, Braatz RD. Mathematical modeling of drug delivery from autocatalytically degradable PLGA micro- spheres-A review. J Control Release 2013;165:29-37.

[29] Mattu C, Pabari RM, Boffito M, Sartori S, Ciardelli G, Ramtoola Z. Comparative evaluation of novel biodegradable nanoparticles for the drug targeting to breast cancer cells. Eur J Pharm Biopharm 2013;85:463-472.

[30] Shah SA, Potter MW, McDade TP, Ricciardi R, Perugini RA, Elliott PJ, Adams J, Callery MP. 26S Proteasome inhibition induces apoptosis and limits growth of human pancreatic cancer. J Cell Biochem 2001;82:110-122.

[31] Bold RJ, Virudachalam S, McConkey DJ. Chemosensitization of pancreatic cancer by inhibition of the 26S proteasome. J Surg Res 2001;100:1117.

[32] Nawrocki ST, Bruns CJ, Harbison MT, Bold RJ, Gotsch BS, Abbruzzese JL, Elliott P, Adams J, McConkey DJ. Effects of the proteasome inhibitor PS341 on apoptosis and angiogenesis in orthotopic human pancreatic tumor xenografts. Mol Cancer Ther 2002;1:1243-1253.

[33] Nawrocki ST, Sweeney-Gotsch B, Takamori R, McConkey DJ. The proteasome inhibitor bortezomib enhances the activity of doce- taxel in orthotopic human pancreatic tumor xenografts. Mol Can- cer Ther 2004;3:5970 .

[34] Nawrocki ST, Carew JS, Dunner K Jr, Boise LH, Chiao PJ, Huang P, Abbruzzese JL, McConkey DJ. Bortezomib inhibits PKR-like endoplasmic reticulum (ER) kinase and induces apoptosis via ER stress in human pancreatic cancer cells. Cancer Res 2005;65: 11510-11519.

[35] Adams J, Palombella VJ, Sausville EA, Johnson J, Destree A, Lazarus DD, Maas J, Pien CS, Prakash S, Elliott PJ. Proteasome inhibitors: A novel class of potent and effective antitumor agents. Cancer Res 1999;59:2615-2622.

[36] Mura S, Hillaireau H, Nicolas J, Le Droumaguet B, Gueutin C, Zanna S, Tsapis N, Fattal E. Influence of surface charge on the potential toxicity of PLGA nanoparticles towards Calu-3 cells. Int J Nanomedicine 2011;6:2591-2605.

[37] Menon JU, Kona S, Wadajkar AS, Desai F, Vadla A, Nguyen KT. Effects of surfactants on the properties of PLGA nanoparticles. J Biomed Mater Res A 2012;100:1998-2005.

[38] Panyam J, Labhasetwar V. Dynamics of endocytosis and exocyto- sis of

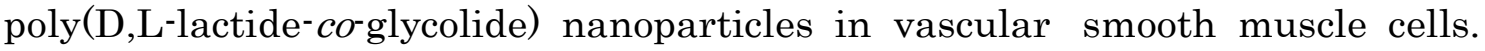
Pharm Res 2003;20:212-220.

[39] Sorokin LM, Morgan EH, Yeoh GCT. Transformation-induced changes in transferrin and iron metabolism in myogenic cells. Cancer Res 1989;49:19411947.

[40] Milani S, Bombelli FB, Pitek AS, Dawson KA, Rædler J. Reversible versus irreversible binding of transferrin to polystyrene nanoparticles: Soft and 
hard corona. ACS Nano 2012;6:2532-2541.

[41] Sempf K, Arrey T, Gelperina S, Schorge T, Meyer B, Karas M, Kreuter J. Adsorption of plasma proteins on uncoated PLGA nanoparticles. Eur J Pharm Biopharm 2013;85:53-60.

[42] Choi CHJ, Alabi CA, Webster P, Davis ME. Mechanism of active targeting in solid tumors with transferrin-containing gold nanoparticles. Proc Natl Acad Sci USA 2010;107:1235-1240.

[43] Salvati A, Pitek AS, Monopoli MP, Prapainop K, Bombelli FB, Hristov DR, Kelly PM, A berg C, Mahon E, Dawson KA. Transferrinfunctionalized nanoparticles lose their targeting capabilities when a biomolecule corona adsorbs on the surface. Nat Nanotechnol 2013;8:137-143.

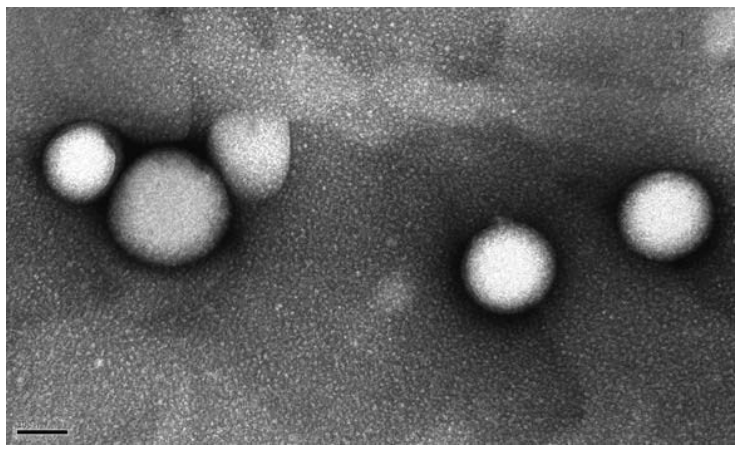

FIGURE 1. TEM image of unloaded PLGA nanoparticles; scale bar: $100 \mathrm{~nm}$.

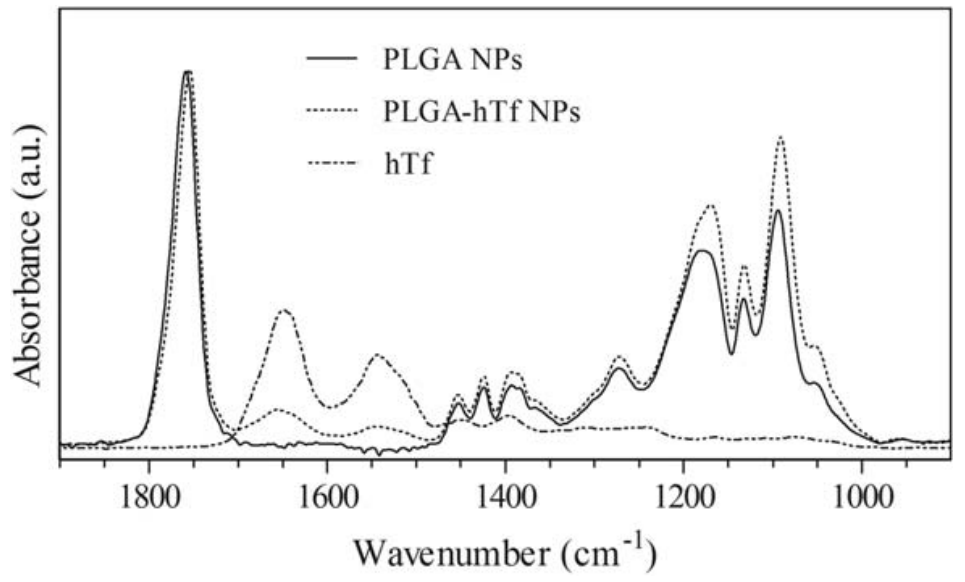

FIGURE 2. ATR-FT-IR spectra of unloaded PLGA nanoparticles (PLGA NPs), holotransferrin (hTf), and PLGA NPs with hTf surface- modification. 


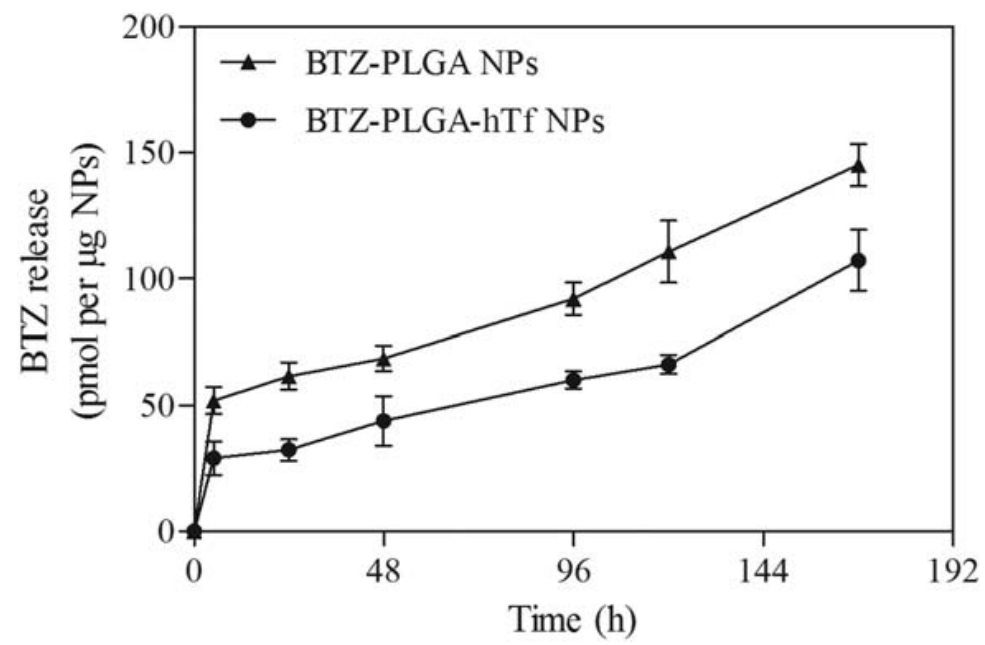

FIGURE 3. In vitro release profiles of bortezomib (BTZ) from PLGA nanoparticles (NPs) and PLGA NPs surface-modified with holotransferrin (hTf), evaluated through the inhibition of the $20 \mathrm{~S}$ proteasome activity. Results are shown as mean 6 SEM of three independent experiments.
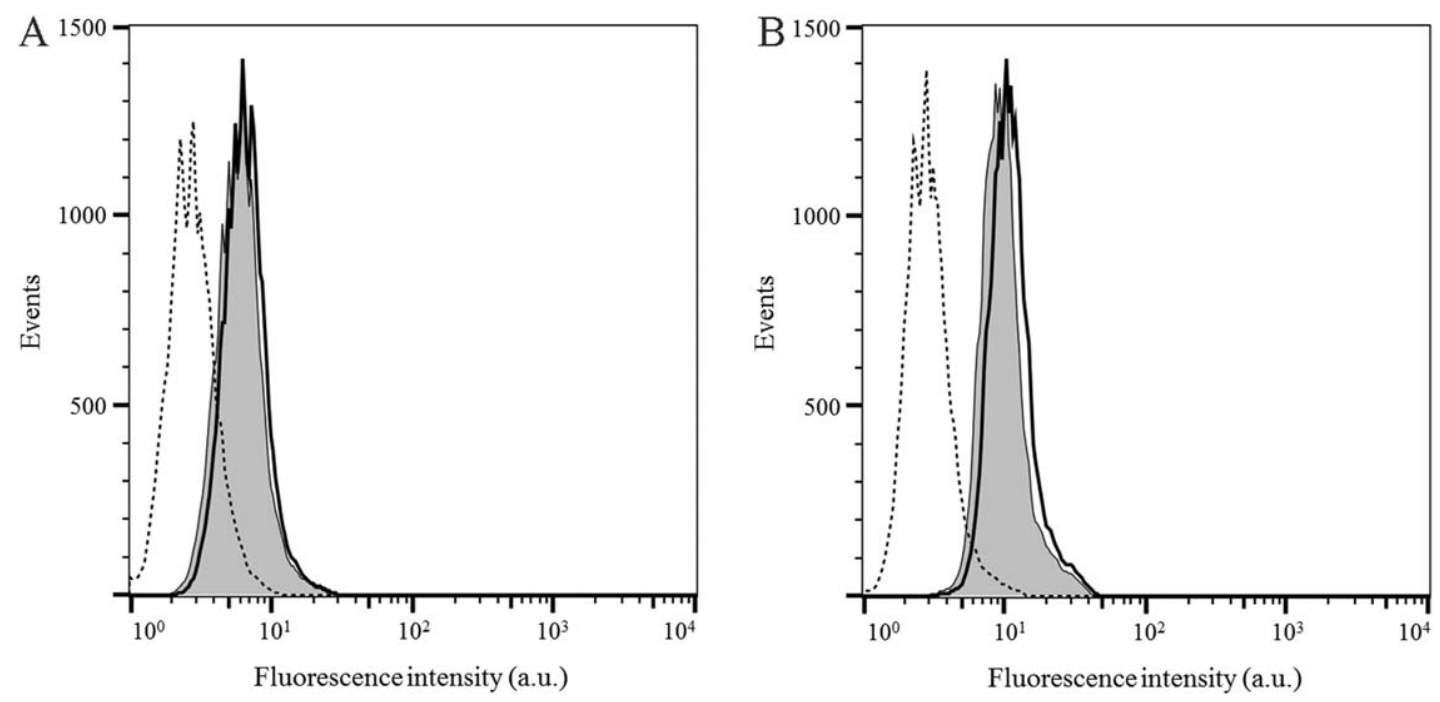

FIGURE 4. Uptake of nanoparticles (NPs) by hTERT-HPNE (A) and S2-013 (B) cells assessed by FCM. The dotted line histogram represents cells stained only with propidium iodide (negative control), the grey area histogram shows cells incubated with coumarin-6 (C6)-PLGA NPs and the black line histogram shows cells incubated with C6-PLGA NPs surface-modified with holo-transferrin. 

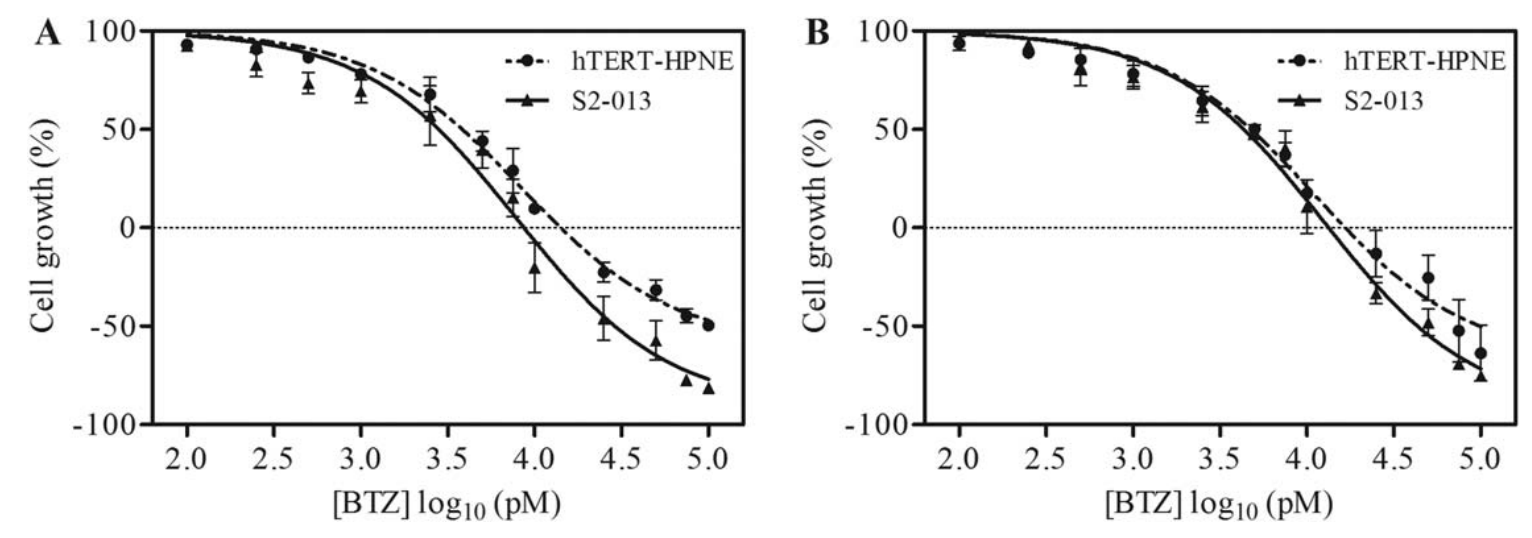

FIGURE 5. Concentration-dependent effect of $48 \mathrm{~h}$ incubation with bortezomib (BTZ) on the growth of hTERT-HPNE and S2-013 cells, evaluated by PrestoBlue VR reagent $(A)$ and sulforhodamine $B$ assay $(B)$. Results are shown as mean 6 SEM of at least three independent experiments.

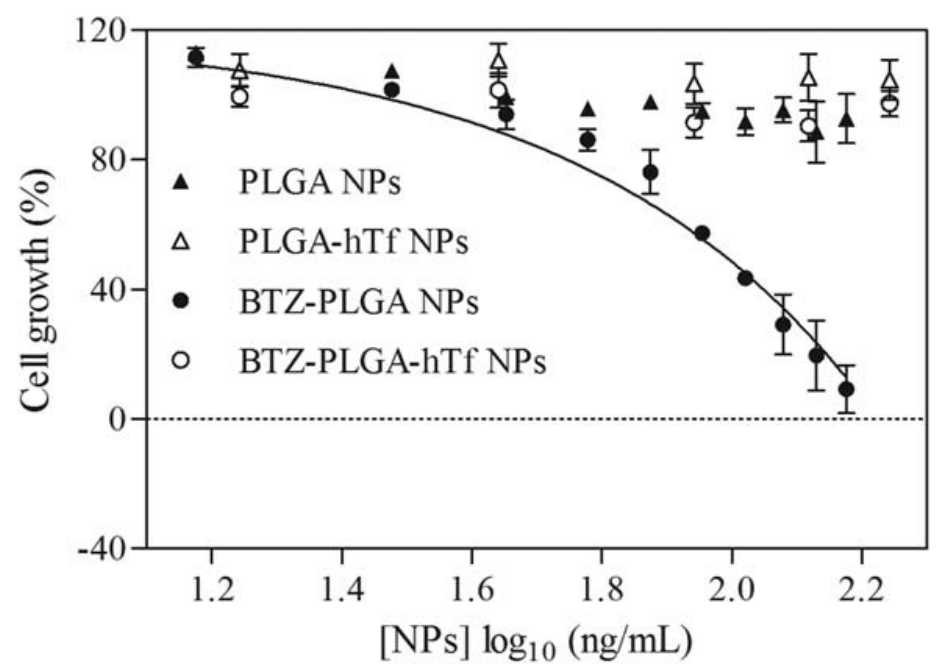

FIGURE 6. Response of hTERT-HPNE cells to $48 \mathrm{~h}$ incubation with nanoparticles.

Cell growth inhibition evaluated by PrestoBlue $\mathrm{R}$ rea-gent. Results are shown as mean 6 SEM of at least three independent experiments. 


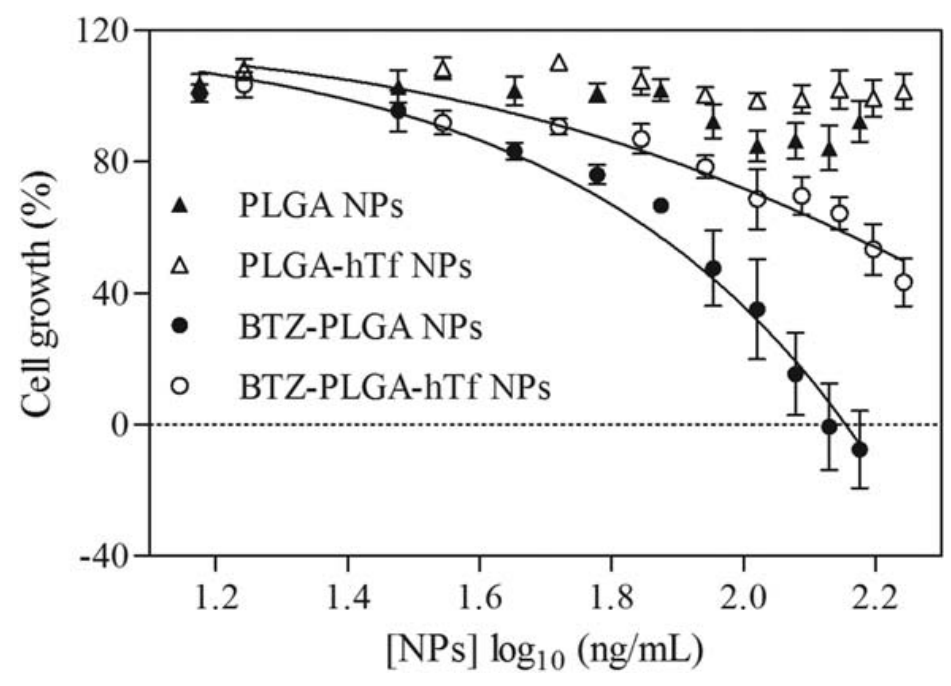

Figure 7. Response of S2-013 cells to $48 \mathrm{~h}$ incubation with nanoparticles Cell growth inhibition evaluated by PrestoBlue ${ }^{\circledR}$ reagent. Results are shown as mean 6 SEM of at least three independent experiments.

TABLE I. Properties of Unloaded PLGA Nanoparticles (PLGA NPs) and Loaded with Bortezomib (BTZ), Both Unmodified and with Holo-Transferrin (hTf) SurfaceModification

\begin{tabular}{lccc}
\hline & $\begin{array}{c}\text { Mean Diameter } \\
(\mathrm{nm})\end{array}$ & $\begin{array}{c}\text { Polydispersity } \\
\text { Index }\end{array}$ & $\begin{array}{c}\zeta \text { Potential } \\
(\mathrm{mV})\end{array}$ \\
\hline PLGA NPs & $192 \pm 14$ & $0.06 \pm 0.02$ & $-27 \pm 4$ \\
BTZ-PLGA NPs & $195 \pm 12$ & $0.06 \pm 0.02$ & $-27 \pm 2$ \\
PLGA-hTf NPs & $198 \pm 8$ & $0.09 \pm 0.03$ & $-14 \pm 1$ \\
BTZ-PLGA-hTf NPs & $200 \pm 10$ & $0.07 \pm 0.05$ & $-16 \pm 1$ \\
\hline
\end{tabular}

Results are shown as mean $\pm \mathrm{SD}$ of at least three independent experiments.

TABLE II. Cytotoxic Effects of Bortezomib to hTERT-HPNE and S2-013 Cells Expressed by the Estimated GI50 Values (Concentrations in nM that cause $50 \%$ Inhibition of Cell Growth) at $48 \mathrm{~h}$ of Treatment

\begin{tabular}{lcc}
\hline & \multicolumn{2}{c}{$\mathrm{GI}_{50}(\mathrm{n} M)$} \\
\cline { 2 - 3 } & $\mathrm{PB}$ & $\mathrm{SRB}$ \\
\hline hTERT-HPNE & $3.7 \pm 0.6$ & $4.5 \pm 1.2$ \\
S2-013 & $2.7 \pm 1.1$ & $4.3 \pm 1.5$ \\
\hline
\end{tabular}

The cytotoxicity was evaluated by PrestoBlue ${ }^{\oplus}$ (PB) and sulforhodamine $B$ (SRB) assays. Results are shown as mean \pm SD of at least three independent experiments. 